\title{
Optimizing Heart Rate Regulation for Safe Exercise
}

\author{
Steven W. Su ${ }^{\dagger *}$, Shoudong Huang ${ }^{\dagger}$, Lu Wang ${ }^{\ddagger}$, Branko G. Celler ${ }^{\ddagger}$, \\ Andrey V. Savkin ${ }^{\ddagger}$, Ying Guo ${ }^{\S}$, and Teddy M. Cheng ${ }^{\ddagger}$
}

\begin{abstract}
Safe exercise protocols are critical for effective rehabilitation programs. This paper aims to develop a novel control strategy for an automated treadmill system to reduce the danger of injury during cardiac rehabilitation. We have developed a control-oriented nonparametric Hammerstein model for the control of heart rate during exercises by using support vector regression and correlation analysis. Based on this nonparametric model, a model predictive controller has been built. In order to guarantee the safety of treadmill exercise during rehabilitation, this new automated treadmill system is capable of optimizing system performance over predefined ranges of speed and acceleration. The effectiveness of the proposed approach was demonstrated with six subjects by having their heart rate track successfully a predetermined heart rate.
\end{abstract}

Keywords: Rehabilitation, Hammerstein model identification, Model Predictive Control, Support Vector Regression, Nonparametric model.

$\dagger$ The Faculty of Engineering and Information Technology, University of Technology, Sydney, Australia.

$\ddagger$ Human Performance Group, Biomedical Systems Lab, School of Electrical Engineering \& Telecommunications University of New South Wales, UNSW Sydney N.S.W. 2052 Australia.

$\S$ Autonomous Systems Lab, CSIRO ICT Center, Australia.

* The corresponding author is with Faculty of Engineering and Information Technology, University of Technology, Sydney, Australia. Email: Steven.Su@uts.edu.au. Tel: +61 (2) 95147603.

This research was supported by the Australian Research Council. 


\section{INTRODUCTION}

An automated exercise system can be useful in providing a platform for the development of exercise protocols suitable for rehabilitation, medical diagnosis, sport training and analysis of cardio respiratory kinetics [1] [9] [24]. In our previous study [29], an automated treadmill exercise system was developed. This system can regulate the heart rate kinetics of the exercising individual according to a prescribed heart rate profile preventing over stressing of the cardiovascular system. However, exercising on a treadmill is not without risks and may result in serious injuries [7] [12] [30]. The major safety concern during treadmill exercise is the subject falling. A fall may occur when the treadmill is operating at relatively high speed and/or acceleration. It has also been reported that many obese patients are unable to start exercising on a treadmill, at speeds as low as $2 \mathrm{~km} / \mathrm{h}$ [10] [16]. In order to ensure safe exercise, the speed and acceleration of the automated treadmill therefore need to be restricted to a suitable range. This study has developed a new nonparametric Hammerstein model-based model predictive control (MPC) approach, which can optimally regulate heart rate under predefined speed and acceleration ranges.

A modest extension of the linear model is the Hammerstein model. The Hammerstein model can be described as a static nonlinear block followed by a dynamic linear system. Hammerstein models may account for nonlinear effects encountered not only in industrial processes [13], but also physiological processes [4] [19] [22] [32]. As far as the amount of prior information about the system is concerned, identification problems are either parametric [23] or nonparametric [17]. In the nonparametric problem, the need of prior information is often less than that of parametric problem. However, the nonparametric model will often provide a better fit to experimental data when pre-determining a model structure is difficult. 


\section{Fig. 1. The proposed nonparametric identification procedure.}

This paper presents a nonparametric identification approach for Hammerstein systems (see Fig. 1.) based on the Support Vector Regression (SVR) [31] [15] and the stochastic method [2]. Specifically, pseudo-random binary sequences (PRBS) experiments are performed to decouple the identification of the linear dynamic part from the nonlinearity. Correlation analysis [21] is used to obtain the impulse and step response of the linear dynamic part. The powerful $\epsilon$-insensitivity SVR approach is adopted to model the nonlinearity.

We established a nonparametric Hammerstein model by using the proposed modelling method. Based on the model, we develop a nonparametric model based MPC for an automated exercise system to ensure safe exercise for rehabilitation purposes. The main advantages of MPC is that it allows us to use the detailed knowledge of a process, in the form of a dynamic model, as an aid to controlling that process within the required constraints [14].

MPC has been well developed for linear systems. However, the complexity of the predictive control problem increases significantly for nonlinear systems. In the case of Hammerstein systems, the most commonly used control method is based on direct inversion of the static nonlinearity combined with existing linear control approaches [20] [29]. This strategy is also adopted in this paper. Firstly, the approximation of the inversion of static nonlinearity is directly obtained by using $\epsilon$-insensitivity SVR. This nonlinearity is used as a pre-compensator to cancel the input nonlinearity. The model predictive controller is then designed for the approximated linear model to achieve desired tracking performance under predefined constraints. Finally, the proposed MPC approach is applied for the automated heart rate regulation system design. This study establishes a unified frame work for identification and control of nonparametric Hammerstein systems for treadmill rehabilitation exercises. Preliminary data from this study, with simulation results only, was published in the proceeding of the EMBS 07 conference [27]. This paper presents the completed study with real time implementation and experimental validation.

The paper is organized as follows. A brief description of the problem is given in Section II. The proposed identification and model predictive control approach are given in Section III. Section IV describes the identification of Hammerstein model of heart rate response for treadmill exercises. Experimental results for the regulation of heart rate of treadmill exercisers are also presented in Section IV. Section V concludes the paper. 


\section{Fig. 2. The automated treadmill system.}

\section{PROBLEM DESCRIPTION}

The problem considered in this study is the development of a controller for an automated treadmill system to provide safe exercise protocols. The potential applications arising from this study include the training of elite athletes, as well as the rehabilitation of patients with cardiac diseases, diabetes and obesity. The experimental settings are shown in Fig.2. The controller's input is the measured heart rate, whereas the output of the controller is the actuating signal that controls the speed of the treadmill.

In our previous study [29], a nonlinear robust control approach was developed and desired tracking performance was achieved under normal working conditions. However, for some cases, the transmission of ECG signal (heart rate variation) was significantly perturbed due to the high impedance of electrodes, electrical and electromagnetic interface (EMI), and accidental disconnection of the electrodes. This often led to a sudden halt or dramatic increase of treadmill speed, compromising the safety of the user.

As stated in the introduction, in order to ensure the safety of rehabilitation exercise, maximum allowable constraints on the speed and acceleration of the treadmill need to be imposed in the controller. The role of the controller is to optimally regulate the heart rate by adjusting the speed of the treadmill under speed and acceleration constraints.

\section{PROPOSED MODELLING AND CONTROL APPROACH}

In this paper, we use a Hammerstein model to dynamically describe the relationship between walking speed and heart rate variation. As mentioned in the introduction, the linear dynamic identification of Hammerstein models can be decoupled from that of nonlinear parts by using PRBS experiments [2]. However, the PRBS inputs often cannot excite the nonlinearity sufficiently. To identify the nonlinear part or its inverse, steady state experiments should be performed. 


\section{Fig. 3. The precompensated system.}

\section{A. Modelling the inverse of the nonlinear function by using SVR}

To transfer a Hammerstein system to a linear system, a pre-compensator (essentially, the inverse of the nonlinearity) can be applied as in [20] and [29] (See Fig.3).

For the identification of the inverse of the nonlinearity, the so called $\epsilon$-insensitivity SVR will be employed, which is convex and very efficient in terms of speed and complexity. Now we briefly introduce the SVR approach [31].

Let $\left\{u_{i}, y_{i}\right\}_{i=1}^{N}$ be a set of inputs and outputs data points $\left(u_{i} \in U \subseteq \mathcal{R}^{d}, y_{i} \in Y \subseteq \mathcal{R}, N\right.$ is the number of points). The goal of the SVR is to find a function $f(u)$ which has the following form

$$
f(u)=w \cdot \phi(u)+b,
$$

where $\phi(u)$ represents the high-dimensional feature spaces which are nonlinearly transformed from $u$. The coefficients $w$ and $b$ are estimated by minimizing the regularized risk function:

$$
\frac{1}{2}\|w\|^{2}+C \frac{1}{N} \sum_{i=1}^{N} L_{\epsilon}\left(y_{i}, f\left(u_{i}\right)\right)
$$

The first term is called the regularized term. The second term is the empirical error measured by $\epsilon$-insensitivity loss function which is defined as:

$$
L_{\epsilon}\left(y_{i}, f\left(u_{i}\right)\right)=\left\{\begin{array}{cl}
\left|y_{i}-f\left(u_{i}\right)\right|-\epsilon, & \left|y_{i}-f\left(u_{i}\right)\right|>\epsilon \\
0, & \left|y_{i}-f\left(u_{i}\right)\right| \leq \epsilon
\end{array}\right.
$$

This defines an $\epsilon$ tube. The radius $\epsilon$ of the tube and the regularization constant $C$ are both determined by user.

By solving the above constrained optimization problem, we have

$$
f(u)=\sum_{i=1}^{N} \beta_{i} \phi\left(u_{i}\right) \cdot \phi(u)+b .
$$

where the coefficients $\beta_{i}$ correspond to each $\left(u_{i}, y_{i}\right)$. The support vectors are the input vectors $u_{j}$ whose corresponding coefficients $\beta_{j} \neq 0$.

By the use of kernels, all necessary computations can be performed directly in the input space, without having to compute the map $\phi(u)$ explicitly. After introducing kernel function $k\left(u_{i}, u_{j}\right)$, 
the above equation can be rewritten as follows:

$$
f(u)=\sum_{i=1}^{N} \beta_{i} k\left(u_{i}, u\right)+b,
$$

For linear support regression, the kernel function is thus the inner product in the input space:

$$
f(u)=\sum_{i=1}^{N} \beta_{i}<u_{i}, u>+b .
$$

For nonlinear SVR, there are a number of kernel functions which have been found to provide good generalization capabilities, such as polynomials, radial basis function (RBF), and sigmoid functions. Brief introduction of SVR regression can be found in papers [29] [28]. Details about SVR, such as the selection of radius $\epsilon$ of the tube, kernel function, and the regularization constant $C$, can be found in [31] [26] [18].

It should be emphasized that, as we need to model the inverse of the nonlinear function $f(u)$, the measured steady state output $y$ (heart rate) will be used as the input data, and the input $u$ (treadmill speed) as the output data.

\section{B. Identification of the linear dynamic part}

When a PRBS input is employed for the identification of the Hammerstein system, as shown in equation (2.3) of [2], the identification can be simplified as a linear identification problem.

A PRBS signal is particularly suitable as an experimental input signal for correlation analysis. A PRBS input has two levels $\left(u(t)=u_{1}\right.$ or $u_{2}$ ) and may switch from one level to the other only at multiples of a constant time interval $T_{s}$. A PRBS is periodic with period $T=T_{s} N$, where $N$ is an integer. In order to avoid nonlinear behavior, the difference of the two levels $\left(u_{1}\right.$ and $u_{2}$ ) of PRBS should be as close as possible. However, it is also required that the output responses under these two levels of inputs should be noticeable different (good signal to noise ratio) to ensure a reasonable parameter estimation results. For the selection of Ts and N, we need to compromise with the complexity of the selected model, response time of the system, noise level, and the total experimental time which the subjects can tolerate. In this study, we select $u_{1}=4 \mathrm{~km} / \mathrm{h}, u_{2}=6 \mathrm{~km} / \mathrm{h}, N=31$, and $T_{s}=15 \mathrm{~s}$. The period of PRBS can be calculated as $T=T_{s} \cdot N=465 \mathrm{~s}=7.75$ minutes. It is sufficient to excite the cardio-respiration systems (more than 5 minutes) and short enough to avoid over stressing the subjects to outside the normal 


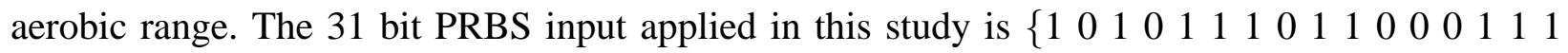
$1100011101000100000\}$ [11].

Correlation analysis [21], which is a nonparametric modelling approach, is applied to identify the step response model of the linear dynamic part in this paper. The step response model is shown as in equation (7) [14]:

$$
y_{k}=\sum_{i=1}^{n-1} h_{i} \Delta u_{k-i}+h_{n} u_{k-n},
$$

where:

- $h_{i}(i=1, \cdots n)$ is the model step response coefficients;

- $n$ is the truncation order;

- $\Delta u_{k}=u_{k}-u_{k-1}$.

It should be noted that the model established in this study is for sample group of healthy young male subjects (aged $31 \pm 5 \mathrm{yr}$, height $176 \pm 5 \mathrm{~cm}$, body weight $74 \pm 11 \mathrm{~kg}$ ).

C. Model predictive controller design

Fig. 4. Model predictive algorithm description.

Our previous work [29] mainly concerns robust performance of heart rate tracking. In this study, in order to ensure the safety of rehabilitation, walking speed and acceleration of the treadmill exercises must be confined to a safe range. MPC is the most suitable selection due to its intrinsic capability of dealing with constraints. After the pre-compensator is employed, the Hammerstein system can be treated as a linear dynamic system. Therefore, linear MPC can be applied to handle this problem. 
MPC predicts and optimizes the future behaviour of the process based on a dynamic model of the process. At each control interval, the MPC algorithm calculates an open-loop sequence of the manipulated variables in such a way as to optimize the future behaviour of the plant [3]. The first value in this optimal sequence is employed into the plant. Fig.4 shows the state of a SISO (single input and single output) MPC system that has been operating for many sampling instants. Integer $k$ represents the current instant. The latest measured output, $y_{k}$, and previous measurements, $y_{k-1}, y_{k-2}, \cdots$, are known.

To calculate its next move $u_{k}$, the controller operates in two phases [3] [25]:

1. Estimation and Prediction: In order to make an intelligent move, the controller needs to know the current state and any internal variables that influence the future trend. To accomplish estimation and prediction, the controller uses all past and current measurements and the models. In this paper, the step response models obtained by using correlation analysis are applied to implement prediction.

2. Optimization: Values of setpoints, measured disturbances, and constraints are specified over a finite horizon of future sampling instants, $k+1, k+2, \cdots, k+p$, where $p$ is the prediction horizon. The controller computes $m$ moves $u_{k}, u_{k+1}, \ldots u_{k+m-1}$, where $m$ is the control horizon. The moves are the solution of a constrained optimization problem:

$$
\min _{\Delta u_{k} \cdots \Delta u_{k+m-1}}\left(\sum_{l=1}^{p}\left\|\hat{y}_{k+l / k}-r_{k+l}\right\|_{\Gamma_{l}^{y}}^{2}+\sum_{l=1}^{m}\left\|\Delta u_{k+l-1}\right\|_{\Gamma_{l}^{u}}^{2}\right),
$$

where:

- $r_{k+l}$ is the targeted output at time $k+l$;

- $\hat{y}_{k+l / k}$ is the predicted values of $y$ at time $k+l$ based on information available at time $k$;

- $p$ is prediction horizon which sets the number of control intervals over which the controller predicts its outputs when computing controller moves;

- $m$ is control horizon which sets the number of moves computed. It must not exceed the prediction horizon. If less than the prediction horizon, the final computed move fills the remainder of the prediction horizon;

- $\|x\|_{\Gamma}^{2}=x^{T} \Gamma x$

- $\Gamma_{l}^{y}$ and $\Gamma_{l}^{u}$ are weighting matrices for predicted errors and control moves $\left(\Gamma_{l}^{y}>0\right.$ and $\Gamma_{l}^{u} \geq 0$ ). For SISO systems, $\Gamma_{l}^{y}$ and $\Gamma_{l}^{u}$ are nonnegative scalars.

Quadratic Programming (QP) was used to minimize the objective function (8). 
FIR (Finite Impulse Response) type models (such as step response models and impulse response models) are the most common models utilised in commercial MPC packages [25]. This is because FIR model based predictions depend only on the input information, as these models have no autoregressive part.

For a step response model, the following form is applied for prediction [3] [25]:

$$
\hat{y}_{k+l / k}=\sum_{i=1}^{n-1} h_{i} \Delta u_{k+l-i}+h_{n} u_{k+l-n}+\hat{d}_{k+l \mid k}
$$

where:

- $\hat{d}_{k+l \mid k}$ is the predicted value of additive disturbance at process output at time $k+l$ based on information available at time $k$ :

$$
\hat{d}_{k+l \mid k}=r(k)-\sum_{i=1}^{n-1} h_{i} \Delta u_{k-i}+h_{n} u_{k+l-n} .
$$

\section{EXPERIMENTS AND DISCUSSIONS}

\section{A. Experimental equipments}

The computer controlled treadmill and its related data collection and processing systems are shown in Fig. 2. The treadmill used in the system is the Powerjog "G" Series fully motorized medical grade treadmill manufactured by Sport Engineering Limited, England. Control of the treadmill can be achieved through an RS232 serial port. The treadmill can receive commands from the computer controller via this link, and obey such commands without supervision. The measurement of heart rate in the designed system is implemented using a wireless Polar system. However, even in the absence of external interference the heart rate can vary substantially over time under the influence of various internal or external factors. Therefore, an improved exponential weighted moving average filter together with a simple outlier detection algorithm is adopted for the estimation of the heart rate. Specifically, the control computer collects heart rate signal from a Polar receiver through an analog input port every 2 seconds, and calculates heart rate by using an edge detection algorithm. Only measured heart rate within a reasonable range (for example, between 50 and 150 beats per minute) was counted in the measured sequence in order to remove outliers.

Assume $\left\{x_{k}\right\}$ is the sequence of the measured heart rate. We apply the exponentially weighted moving average filter to the sequence $\left\{x_{k}\right\}$ :

$$
\bar{x}_{k+1}=\alpha \bar{x}_{k}+(1-\alpha) x_{k} .
$$


where $\alpha$ is the filter coefficient.

\section{B. Experimental procedure and system modelling}

In order to excite nonlinearity sufficiently, steady state experiments are performed and recorded. Six male subjects volunteered to participate in the study (aged $31 \pm 5 \mathrm{yr}$, height $176 \pm 5 \mathrm{~cm}$, body weight $74 \pm 11 \mathrm{~kg}$ ).

All experiments were conducted in the afternoon, and the subjects were permitted to have a light meal one hour before their experiment. Initially, the subjects were asked to walk for about 10 minutes on the treadmill to familiarize themselves with the experiment. The subjects were then requested to walk at five levels of different speeds $(3 \mathrm{~km} / \mathrm{h}, 4 \mathrm{~km} / \mathrm{h}, 5 \mathrm{~km} / \mathrm{h}, 6 \mathrm{~km} / \mathrm{h}$ and a subject specific maximum walking speed, typically $7 \mathrm{~km} / \mathrm{h}$ ). Each level took a total period of 5 minutes, and was followed by a 10-minute resting period. For moderate aerobic exercise, the heart rate normally takes less than 5 minutes to reach steady state and less than 10 minutes to recover (see Figure 1 in [8]). Finally, in order to identify the linear dynamic part of the Hammerstein system, subjects were also requested to walk on the treadmill under a PRBS input.

Fig. 5. Inversion of nonlinearity modelling by using $\epsilon$-insensitivity SVR.

This study applies $\epsilon$-insensitivity SVR regression method to model the inverse of the nonlinear function. The regression result is shown in Fig. 5 where the continuous curve stands for the estimated input-output steady state relationship. The dotted lines indicate the $\epsilon$-insensitivity tube. The plus markers are the points of input and output data. The circled '+' markers are the support points. The selected parameters of the SVR regression are listed as follows. The kernel function is a $\mathrm{RBF}$ function with $\sigma=20.2$. The regularization constant $C$ is equal to 5 . The insensitivity region $\epsilon$ is $0.8 \mathrm{~km} / \mathrm{h}$. The obtained SVR model uses $16.7 \%$ (5 support vectors) of the total 
points available to describe the nonlinear behaviour (with a RMS error $0.5 \mathrm{~km} / \mathrm{h}$ ). The identified static nonlinear inverse model is given as follows:

$$
f(u)=\sum_{i=1}^{N} \beta_{i} k\left(u_{i}, u\right)+b=\sum_{i=1}^{5} \beta_{i} e^{-\frac{\left(u_{i}-u\right)^{2}}{2 \sigma^{2}}}+b,
$$

where, $\beta_{1 \sim 5}=-1.95,-5,5,1.47,0.72, u_{1 \sim 5}=80.5,82,82,106,122$, and $b=5.01$.

For the dynamic modelling of heart rate variation during exercise, a number of parametric models [6] have been proposed based on physiological analysis. However, because of the complexity of physiological responses it may not be appropriate to describe the response of the human cardiovascular system to exercise by a fixed model structure. This paper applies the nonparametric modelling approach based on correlation analysis [21], to model the linear dynamic part of heart rate response.

Fig. 6. Correlation analysis results of six subjects. Top: Step responses of all six subjects based on correlation analysis. Bottom: Normalized step responses of all subjects.

Using experimental data, correlation analysis is then performed. The identified step response for six subjects are shown in Fig.6. The coefficients $h_{1-31}$ in equation (7) is provided as follows: $h_{1-31}=0.0595,0.1516,0.2106,0.2826,0.3332,0.3721,0.4021,0.4431,0.5017,0.5553$, $0.6047,0.6555,0.7256,0.7792,0.8180,0.8488,0.8781,0.8979,0.9205,0.9357,0.9513$, 0.9674, 0.9873, 0.9998, 1.0051, 1.0122, 1.0121, 1.0126, 1.0042, 1.0052, 1.0000 .

\section{MPC control with selected acceleration constraints}

As the inverse of the static nonlinearity has been identified and applied as a precompensator, the compensated system can be regarded as a linear dynamic system (described by a step response model). The optimization problem associated with the MPC controller design is then described 
in equation (8). Before implementing the control system, we need to focus on the definition of system constraints and the selection of prediction and control horizons.

This study investigates constraint selection under the consideration of safety of rehabilitation exercises. The sampling period of the control system is selected as 6 seconds. Treadmill speed is confined between $2 \mathrm{~km} / \mathrm{h}$ and $6.5 \mathrm{~km} / \mathrm{h}$, which is a comfortable and safe walking range for patients. The selection of the constraints for acceleration is also crucial for the safety of old or weak patients due to their relatively slow physical responses. We selected and tested the following three acceleration constraints:

$$
\begin{array}{llll}
\text { a) High: } & {[-4.90,4.90]} & \left(\mathrm{km} \mathrm{h}^{-1} \mathrm{~min}^{-1}\right) \text {; } \\
\text { b) Medium : } & {[-1.68,1.68]} & \left(\mathrm{kmh} \mathrm{h}^{-1} \mathrm{~min}^{-1}\right) \text {; } \\
\text { c) Low : } & {[-0.96,0.96]} & \left(k m h^{-1} \mathrm{~min}^{-1}\right) .
\end{array}
$$

The typical responses for High, Medium and Low acceleration constraints are shown in Fig.7. From Fig.7, we can see that the higher the permitted acceleration range the faster the step responses. However, the differences are not pronounced. For rehabilitation purposes, it is essential that we pay more attention to the safety of the user rather than a faster response.

Fig. 7. Step response under different acceleration constraints. Top: Heart rate sensor output (controlled output). Bottom: Treadmill speed (control effort).

As the wireless heart rate signal is subject to electromagnetic interference and motion artefacts caused by body movements, sensor output may dramatically change (see sensor malfunction A and B in Fig.8). If the acceleration range is High, the automated treadmill system may produce sudden abrupt motions (the circled part of solid line in the bottom of Fig.8). On the other hand, a Low acceleration range can well prevent abrupt motions thus avoiding injury. 
Fig. 8. System response for sensor malfunction under different accelerations.

As a tradeoff between system response and patient safety, we select a Medium acceleration. The overall system constraints are then as follows

$$
\left\{\begin{array}{l}
2 \mathrm{~km} / \mathrm{h} \leq v \leq 6.5 \mathrm{~km} / \mathrm{h} \\
-1.68 \mathrm{~km} \cdot \mathrm{h}^{-1} \cdot \mathrm{min}^{-1} \leq a \leq 1.68 \mathrm{~km} \cdot \mathrm{h}^{-1} \cdot \mathrm{min}^{-1}
\end{array}\right.
$$

It should be emphasized that the proposed speed and acceleration constraints can be further restricted for some special rehabilitation exercises. For example, for the rehabilitation of older patients with cardiac disease, the speed should be confined between $2 \mathrm{~km}$ and $4.5 \mathrm{~km}$, and acceleration should be further confined between $-1 \mathrm{~km} \cdot \mathrm{h}^{-1} \cdot \mathrm{min}^{-1}$ and $1 \mathrm{~km} \cdot \mathrm{h}^{-1} \cdot \mathrm{min}^{-1}$. For other special purpose rehabilitation exercises, constraints can be set in consultation with the patient's clinicians and exercise therapist.

There are no specific rules for the selection of prediction horizon $p$ and control horizon $m$. However, increasing $p$ often results in less aggressive control action, whereas increasing $m$ makes the controller more aggressive and increases computational effort. After extensive simulation and experimental studies, the value of prediction horizon $p$ and control horizon $m$ were determined as 35 and 5 respectively. We also extensively investigated the selection of weighting for predicted errors and control moves. The following values were finally selected: $\Gamma_{l}^{y}=2.2$ and $\Gamma_{l}^{u}=0.9$.

Fig. 9. Experimental results for all six subjects. 
Fig. 10. Comparison between proposed control approach (under different constraints) and $H_{\infty}$ control approach (without constraints).

Experimental results for all six subjects are shown in Fig 9. The control system can reach to target heart rate in less than 130 seconds and without steady state error ${ }^{1}$ for all six subjects. These results are comparable with those obtained in our previous work [29] which applied the $H_{\infty}$ control strategy (see Fig. 10). However, it should be pointed out that the results obtained by the proposed MPC controller are achieved under predefined constraints (13) designed to guarantee safe exercise for rehabilitation.

\section{Fig. 11. A typical experimental result.}

Furthermore, our experimental results show that MPC based control strategy can optimize the control effort by using the model predictive features of the controller as shown in Fig.11. In this example, the control effort (treadmill speed) stops increasing at $t=24.5$ seconds, whereas the heart rate is still far from the set point. This shows that MPC can correctly determine the control effort required to avoid an overshoot based on the model prediction function.

In this study we assume the system is a Hammerstein system. It may not be true for real physiological system. However, the caused modeling error can be handled by the MPC controller. From our experimental results, we also find that this MPC based control approach is robust in the presence of substantial inter subject variability as the desired heart rate responses were achieved for all six subjects. For general purpose exercise training, the group model appears to be adequate and can be used without significant loss of tracking performance. However, in

\footnotetext{
${ }^{1}$ As heart rate is variable even at rest, we consider that the steady state error is zero if the heart rate oscillates around the reference input by no more than $3 \mathrm{bpm}$.
} 
order to further improve control performance and guarantee subject safety during special rehabilitation exercise or high performance athletic training, we would recommend that the group model be recalculated for these specific target groups or even individuals.

\section{CONCLUSION}

One of the main purposes of the developed strategy is to achieve safe exercise protocols in cardiac rehabilitation programs both for normal operating conditions and in the event of sensor and/or actuator malfunction (or failure) [5]. In this paper we propose a Hammerstein modelbased model predictive control approach to achieve accurate control of the heart rate response during exercise. Experimental results show that the proposed control algorithm achieves desired heart rate tracking performance under predefined speed and acceleration constraints and thus can ensure the safety of cardiac rehabilitation exercise. It should be noted that this approach can easily accommodate of actuator failures by simply adding extra control constraints.

In this study, six young and healthy subjects participated in the experimental tests. To further generalize and demonstrate the validity of this approach, this study will be extended to cardiac patients as well as those who are obese or overweight. 


\section{REFERENCES}

[1] A.S. ALeon, B.A. Franklin, and F. Costa et al. Cardiac rehabilitation and secondary prevention of coronary heart disease. Circulation, 111:369-376, 2005.

[2] E.W. Bai. Decoupling the linear and nonlinear parts in Hammerstein model identification. Automatica, 40:671-676, 2004.

[3] Alberto Bemporad, Manfred Morari, and N. Lawrence Ricker. Model Predictive Control Toolbox. The MathWorks, Inc, 1994.

[4] L. Bernotas, P. Crago, and H. Chizeck. A discrete-time model of electrically stimulated muscle. IEEE Trans. Biomed. Eng., 33:829-838, 1986.

[5] M. Blanke, M. Kinnaert, J. Lunze, and M. Staroswiecki. Diagnosis and Fault-Tolerant Control. Springer, Berlin, 2003.

[6] V. Brodan, M. Hajek, and E. Khun. An analog model of pulse rate during physical load and recovery. Physiol. Bohemoslov, 20(2):189, 1971.

[7] C.A. Carmen and B. Chang. Treadmill injuries to the upper extremity in pediatric patients (abstract). Presented at the Pennsylvania Orthopedic Society Spring 2000 meeting, 2000.

[8] Y.L. Chen and Y.H. Lee. Effect of combined dynamic and static workload on heart rate recovery cost. Ergonomics, 41(1):29-38, 1998.

[9] T.M. Cheng, A.V. Savkin, B.G. Celler, S.W. Su, and L. Wang. Nonlinear modelling and control of human heart rate response during exercise with various work load intensities. IEEE Trans. Biomed. Eng., 55(11):2499-2508, 2008.

[10] R.L. Cleland, W.C. Gross, L.D. Koss, M. Daynard, and K.M. Muoio. Weight-loss advertising: An analysis of current trends. A Federal Trade Commission staff report, 2002.

[11] W.D.T. Davies. System Identification for Self-Adaptive Control. Wiley-Interscience, New York, 1970.

[12] J.C. DeToledo and M.R. Lowe. Treadmill injuries in patients with epilepsy. Epilepsy Behav, 4:553-555, 2003.

[13] E. Eskinat, S.H. Johnson, and W.L. Luyben. Use of Hammerstein models in identification of nonlinear systems. AIChE $J ., 37: 255-268,1991$.

[14] C.E. Garcia, D.M. Prett, and M. Morari. Model predictive control: Theory and practice-a survey. Automatica, 25:335-348, 1989.

[15] I. Goethals., K. Pelckmans, J.A.K. Suykens, and B. De Moor. Identification of MIMO Hammerstein models using least squares support vector machines. Automatica, 41:1263-1272, 2005.

[16] L.A. Gondoni, A. Liuzzi, A.M. Titon, O. Taronna, F. Nibbio, P. Ferrari, and G. Leonetti. A simple tool to predict exercise capacity of obese patients with ischaemic heart disease heart. Heart, 92(7):553-555, 2006.

[17] Wlodzimierz Greblicki. Stochastic approximation in nonparametric identification of Hammerstein systems. IEEE Trans. Automat. Contr., 47(11):1800-1810, 2002.

[18] Y. Guo, P.L. Bartlett, J. Shawe-Taylor, and R.C. Williamson. Covering numbers for support vector machines. IEEE Trans. on Information Theory, 48(1):239-250, 2002.

[19] K.J. Hunt, M. Munih, N.de N. Donaldson, and F.M.D. Barr. Investigation of the Hammerstein hypothesis in the modeling of electrically stimulated muscle. IEEE Trans. Biomed. Eng., 45(8):998-1009, 1998.

[20] G.A. Ingram, M.A. Franchek, V. Balakrishnan, and G. Surnilla. Robust SISO $\mathcal{H}_{\infty}$ controller design for nonlinear systems. Control Engineering Practice, 13:1413-1423, 2005.

[21] L. Ljung. System Identification: Theory for the user. Prentice-Hall, Englewood Cliffs, 1987.

[22] G.N. Maksym, R.E. Kearney, and J.H. Bates. Nonparametric block-structured modeling of lung tissue strip mechanics. Ann Biomed Eng, 26(2):242-252, 1998. 
[23] K.S. Narendra and P.G. Gallman. An iterative method for the identification of nonlinear systems using Hammerstein model. IEEE Trans. Automat. Contr., 11:546-550, 1966.

[24] A.L. Ries, G.S. Bauldoff, B.W. Carlin, R. Casaburi, C.F. Emery, D.A. Mahler, B. Make, C.L. Rochester, R. ZuWallack, and C. Herrerias. Pulmonary rehabilitation: Joint accp/aacvpr evidence-based clinical practice guidelines. Chest, 131:4-42, 2007.

[25] J.A. Rossiter. Model-based Predictive Control. CRC PRESS, London, 2003.

[26] B. Schlkopf and A. Smola. Learning with kernels. MA: MIT Pres, Cambridge, 2002.

[27] S.W. Su, S. Huang, L. Wang, B.G. Celler, A.V. Savkin, Y. Guo, and T. Cheng. Nonparametric Hammerstein model based model predictive control for heart rate regulation. Proc. IEEE EMBS, pages 2984-2987, Lyon, France, 23-26 Aug, 2007.

[28] S.W. Su, L. Wang, B.G. Celler, and A.V. Savkin. Oxygen uptake estimation in humans during exercise using a Hammerstein model. Ann. Biomed. Eng., 35(11):1898-1906, 2007.

[29] S.W. Su, L. Wang, B.G. Celler, A.V. Savkin, and Y. Guo. Identification and control for heart rate regulation during treadmill exercise. IEEE Trans. Biomed. Eng., 54(7):1238-1246, 2007.

[30] US Consumer Product Safety Commission. Sports-related injuries to older americans. Washington, DC: Office of Information and Public Affairs, 1998.

[31] V. Vapnik. Statistical Learning Theory. John Wiley, New York, 1998.

[32] G. Wilhere, P. Crago, and H. Chizeck. Design and evaluation of a digital closed-loop controller for the regulation of muscle force by recruitment modulation. IEEE Trans. Biomed. Eng., 32:668-676, 1985. 


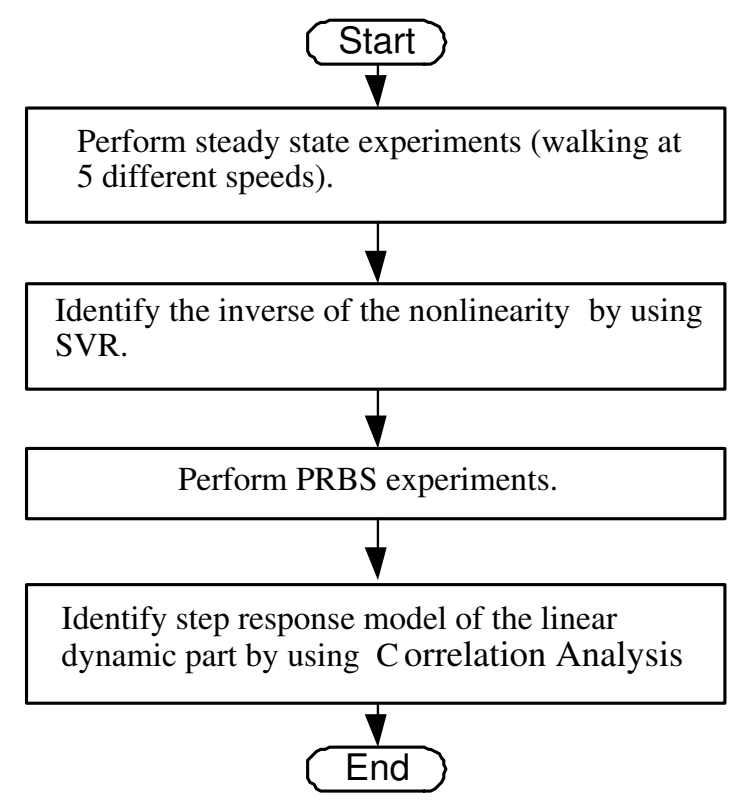

Fig. 1. The proposed nonparametric identification procedure. 

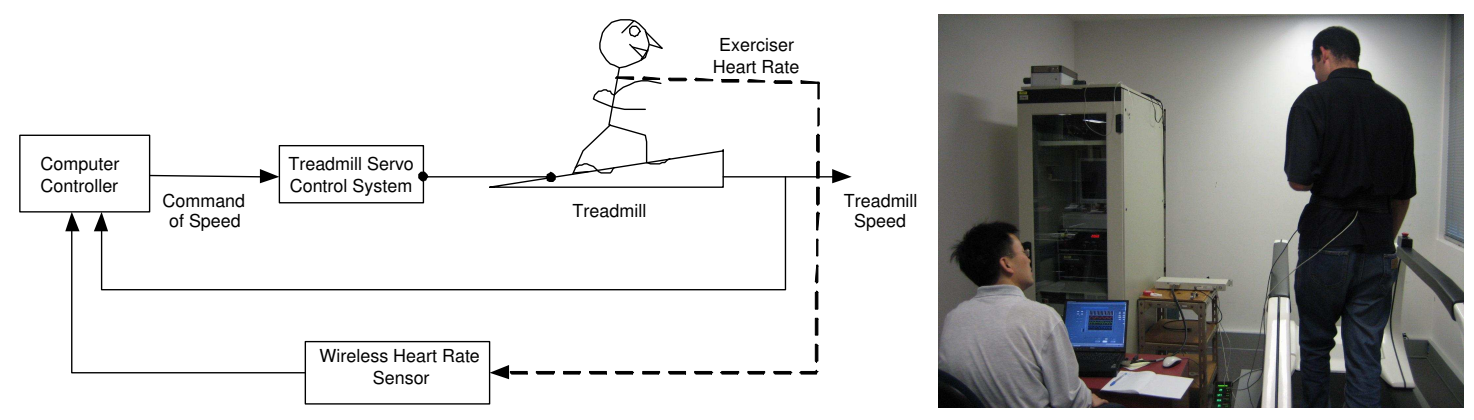

Fig. 2. The automated treadmill system. 
Approximated linear system by pre-compensation

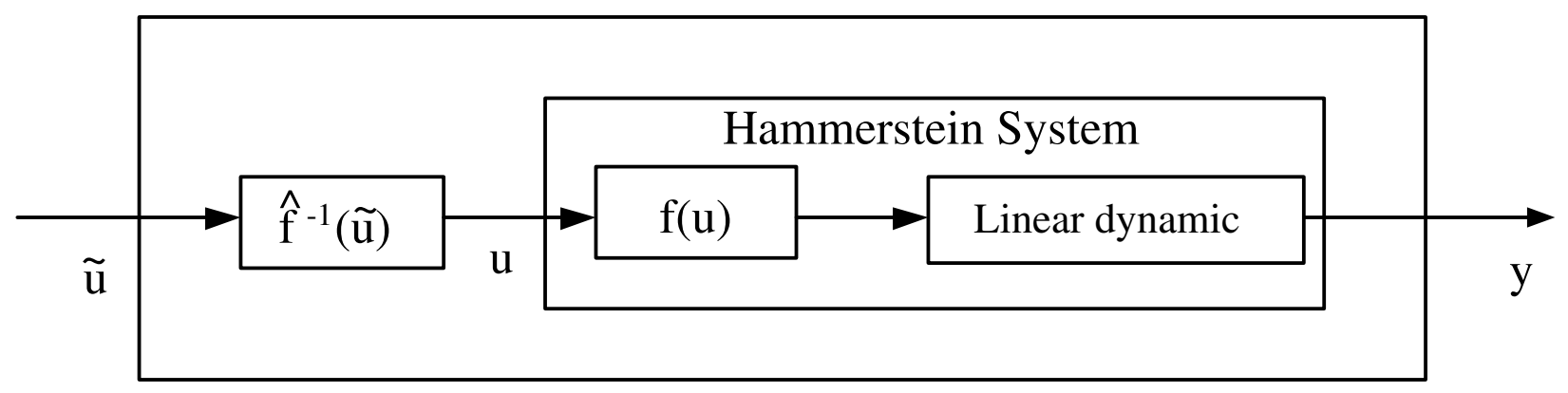

Fig. 3. The precompensated system. 


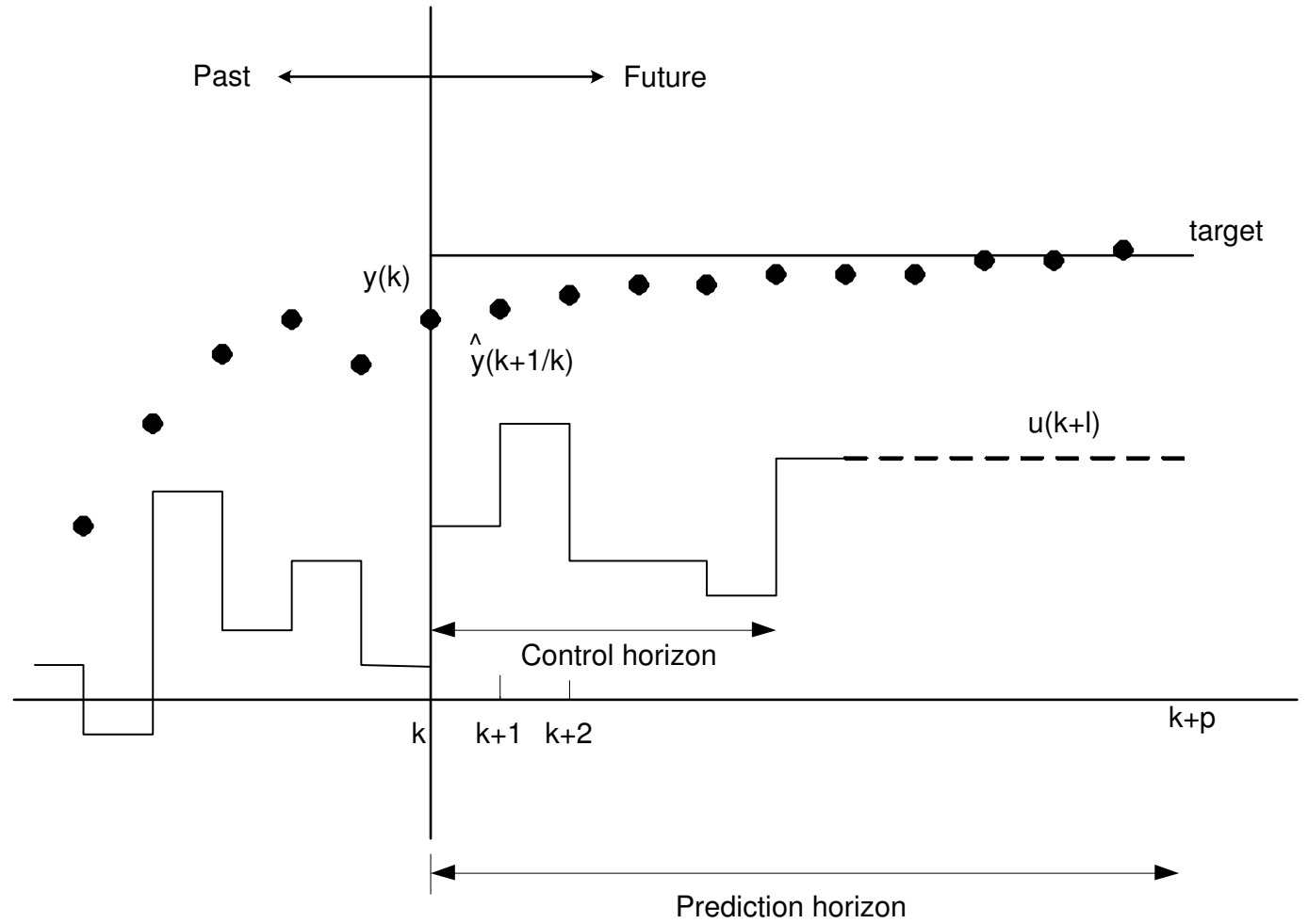

Fig. 4. Model predictive algorithm description. 


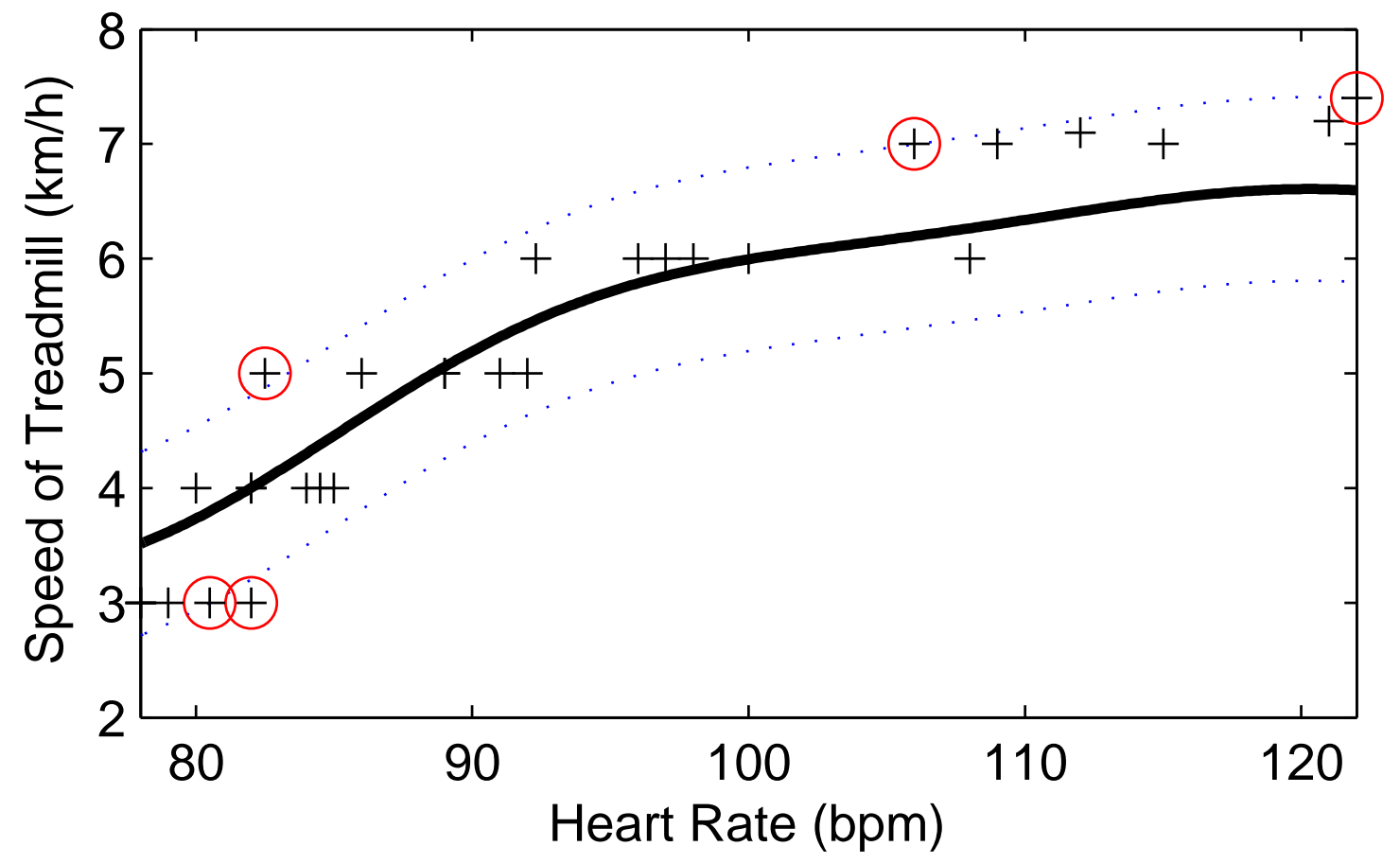

Fig. 5. Inversion of nonlinearity modelling by using $\epsilon$-insensitivity SVR 

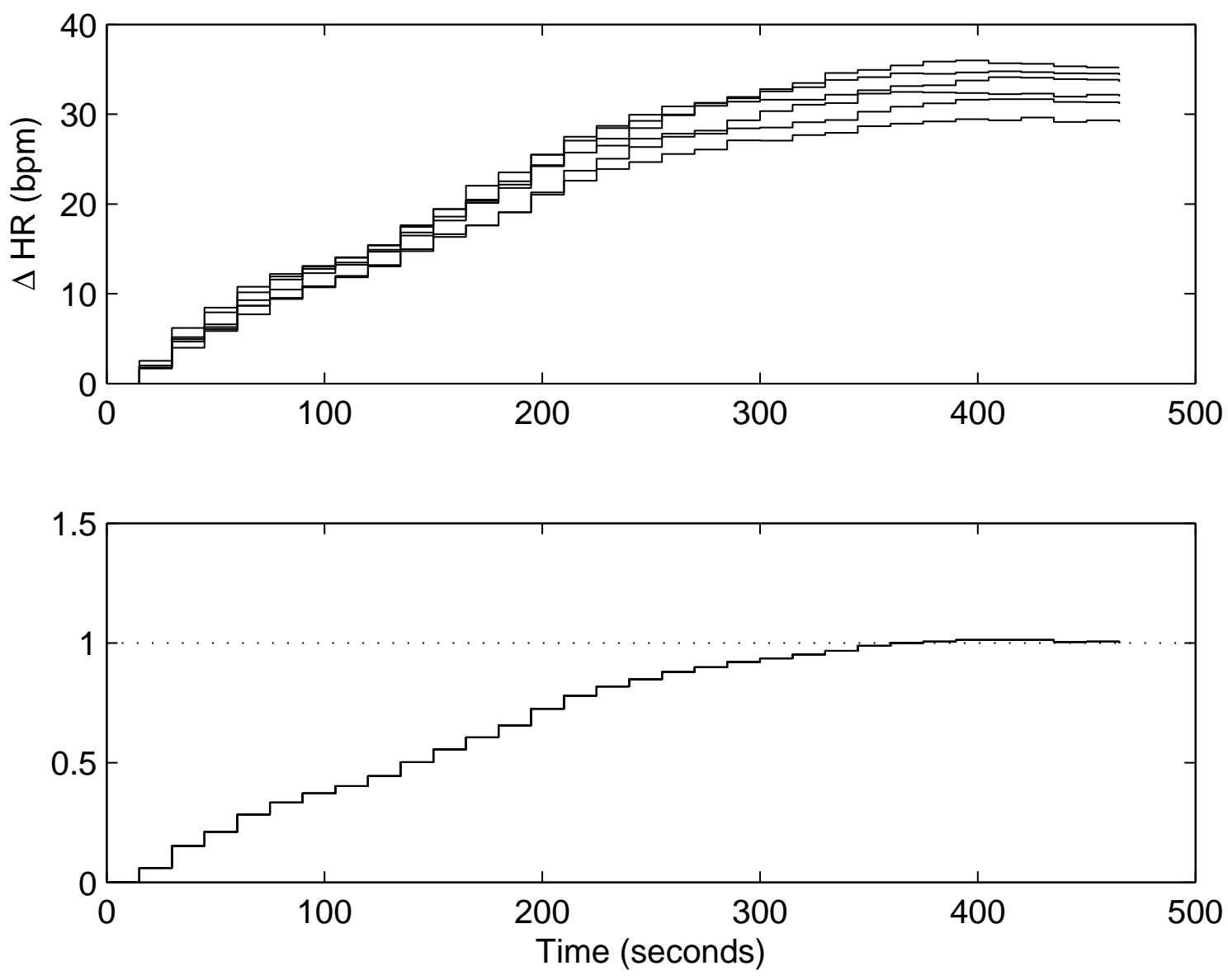

Fig. 6. Correlation analysis results of six subjects. Top: Step responses of all six subjects based on correlation analysis. Bottom: Normalized step responses of all subjects. 

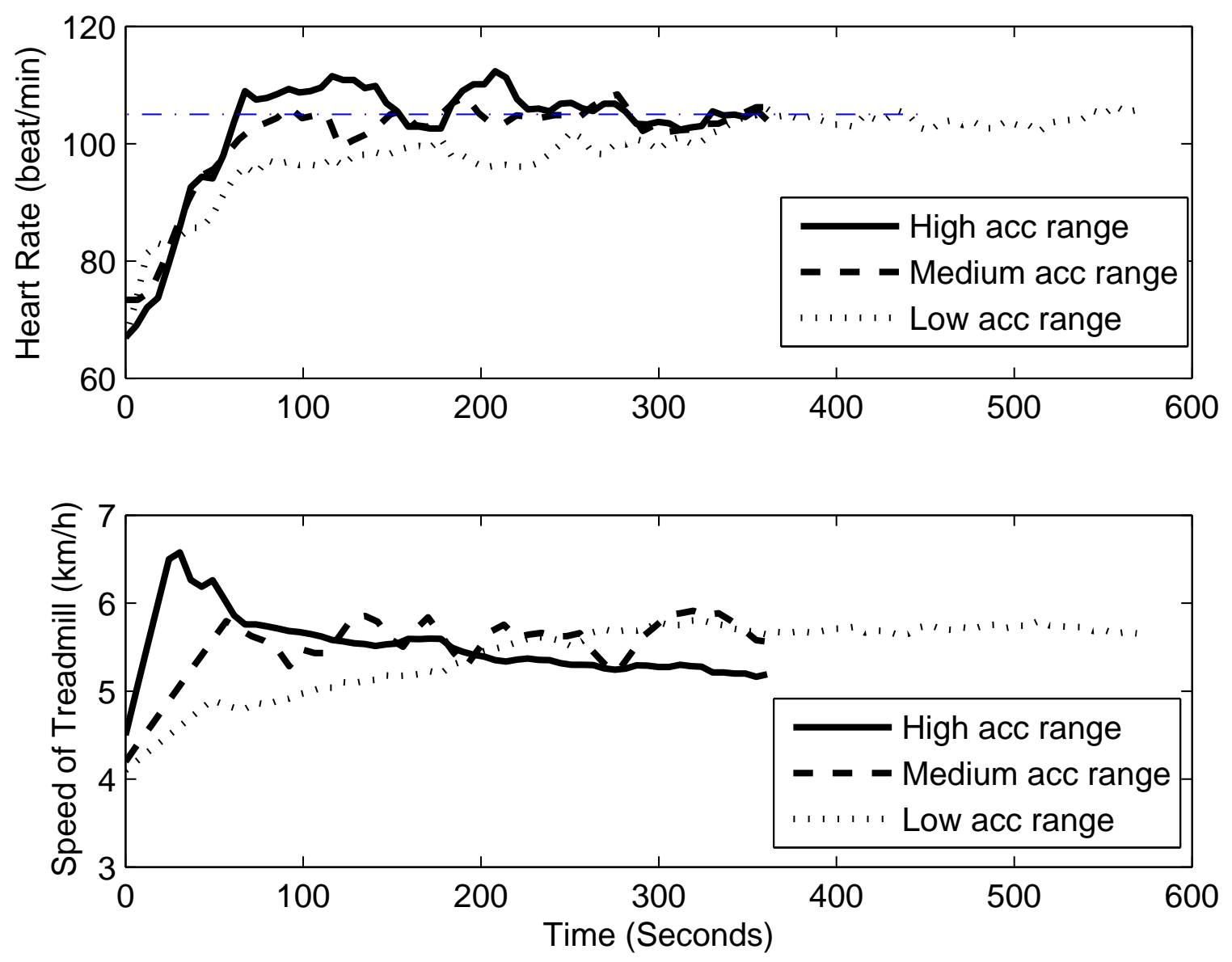

Fig. 7. Step response under different acceleration constraints. Top: Heart rate sensor output (controlled output). Bottom: Treadmill speed (control effort). 

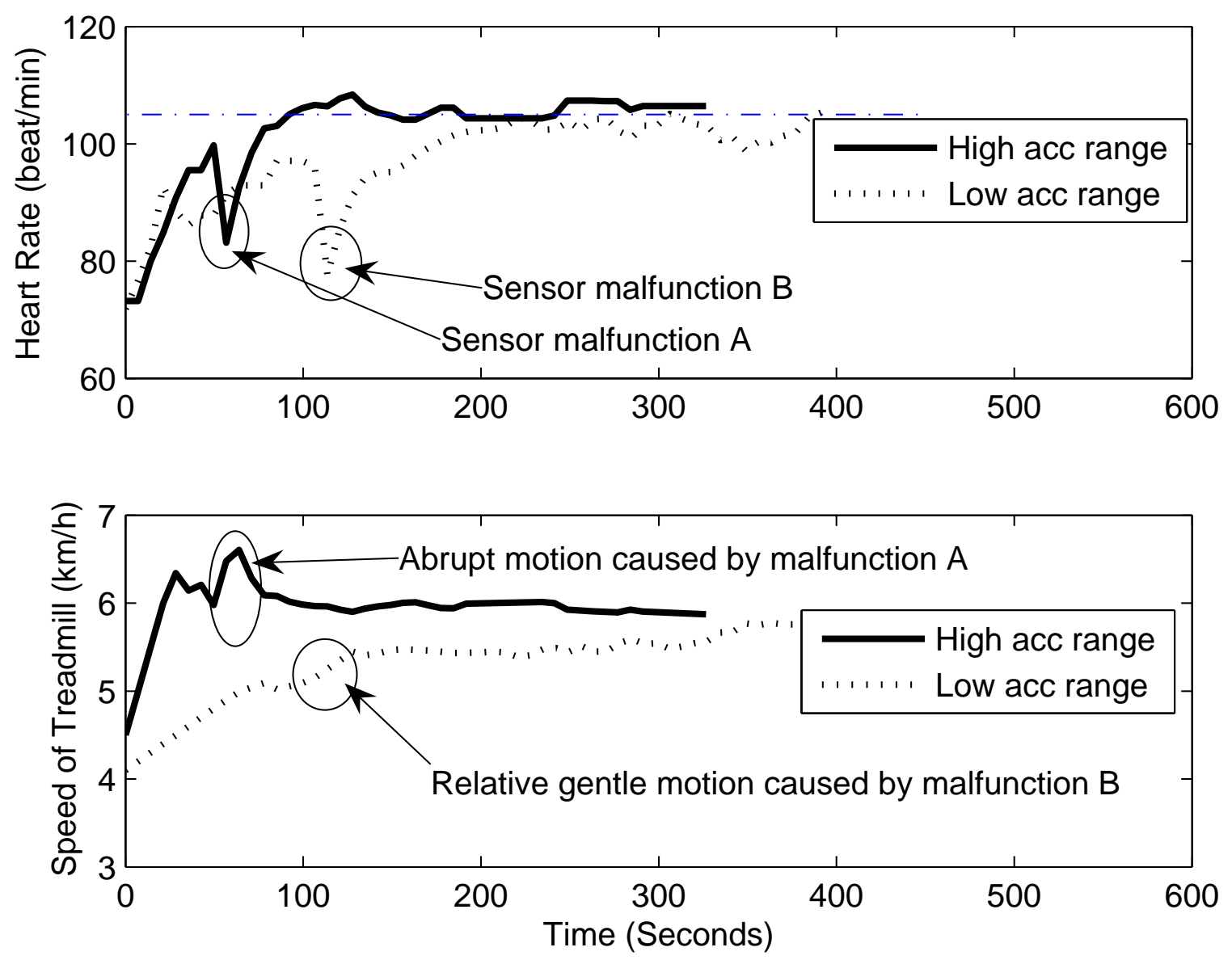

Fig. 8. System response for sensor malfunction under different accelerations. 

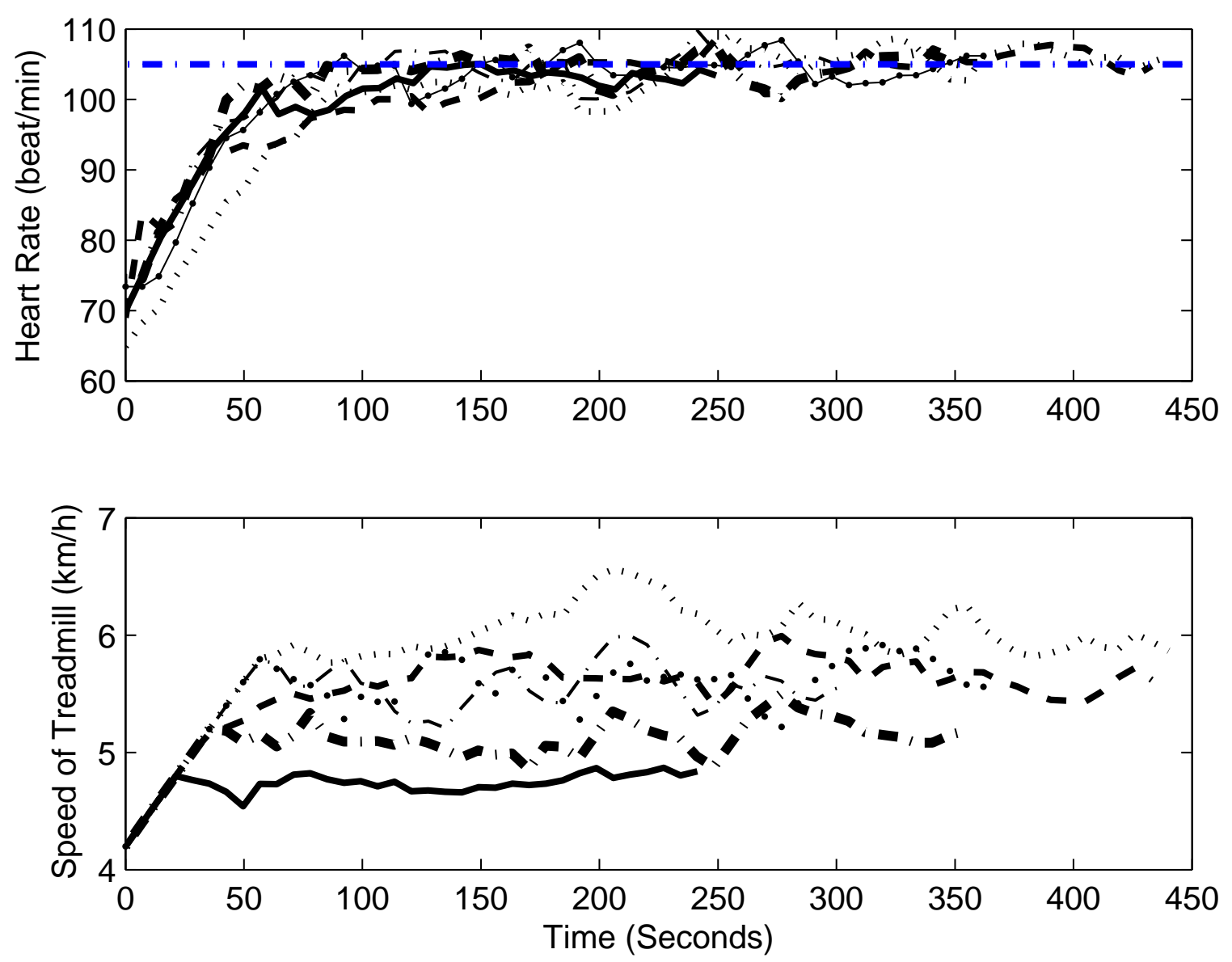

Fig. 9. Experimental results for all six subjects. 

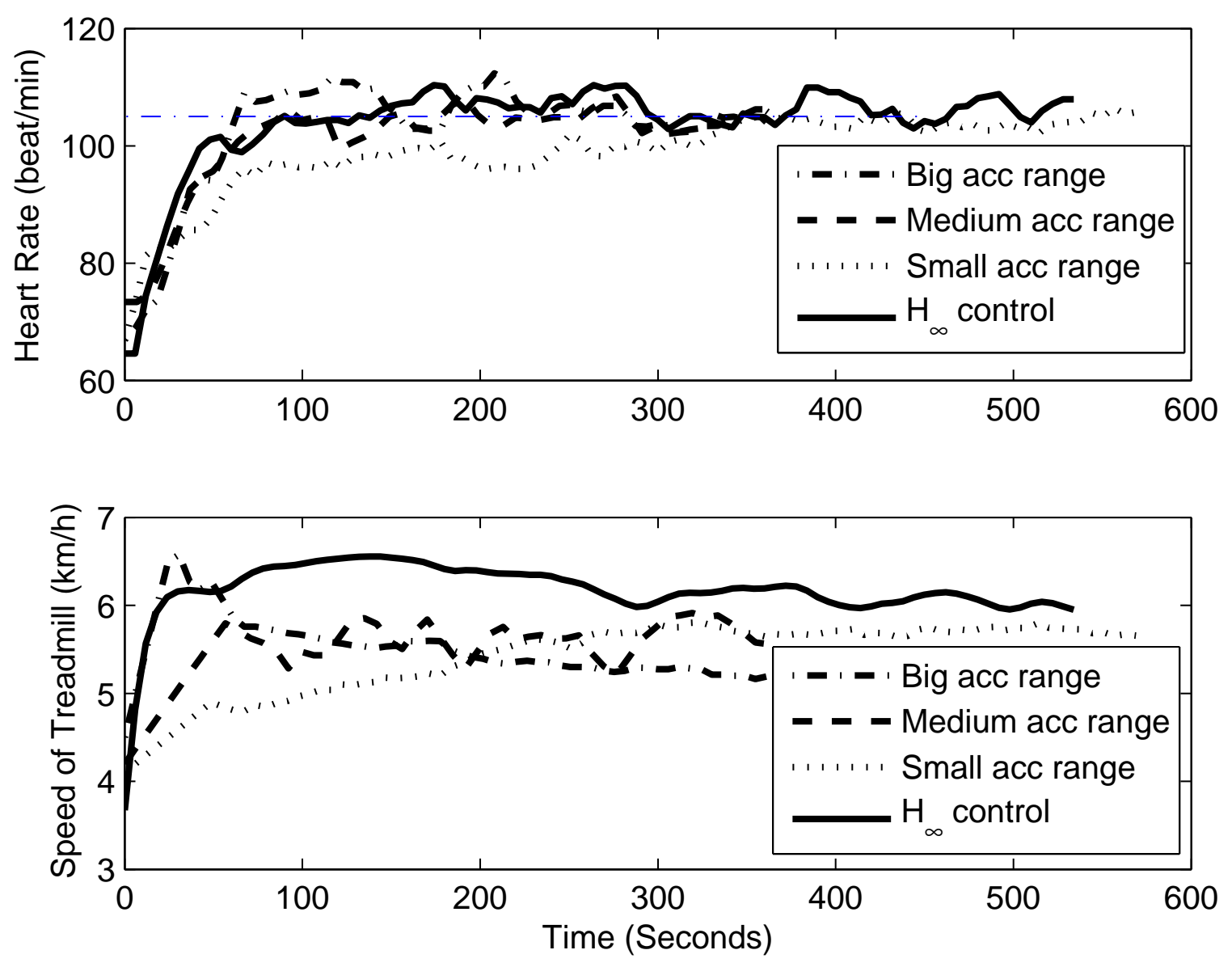

Fig. 10. Comparison between proposed control approach (under different constraints) and $H_{\infty}$ control approach (without constraints). 


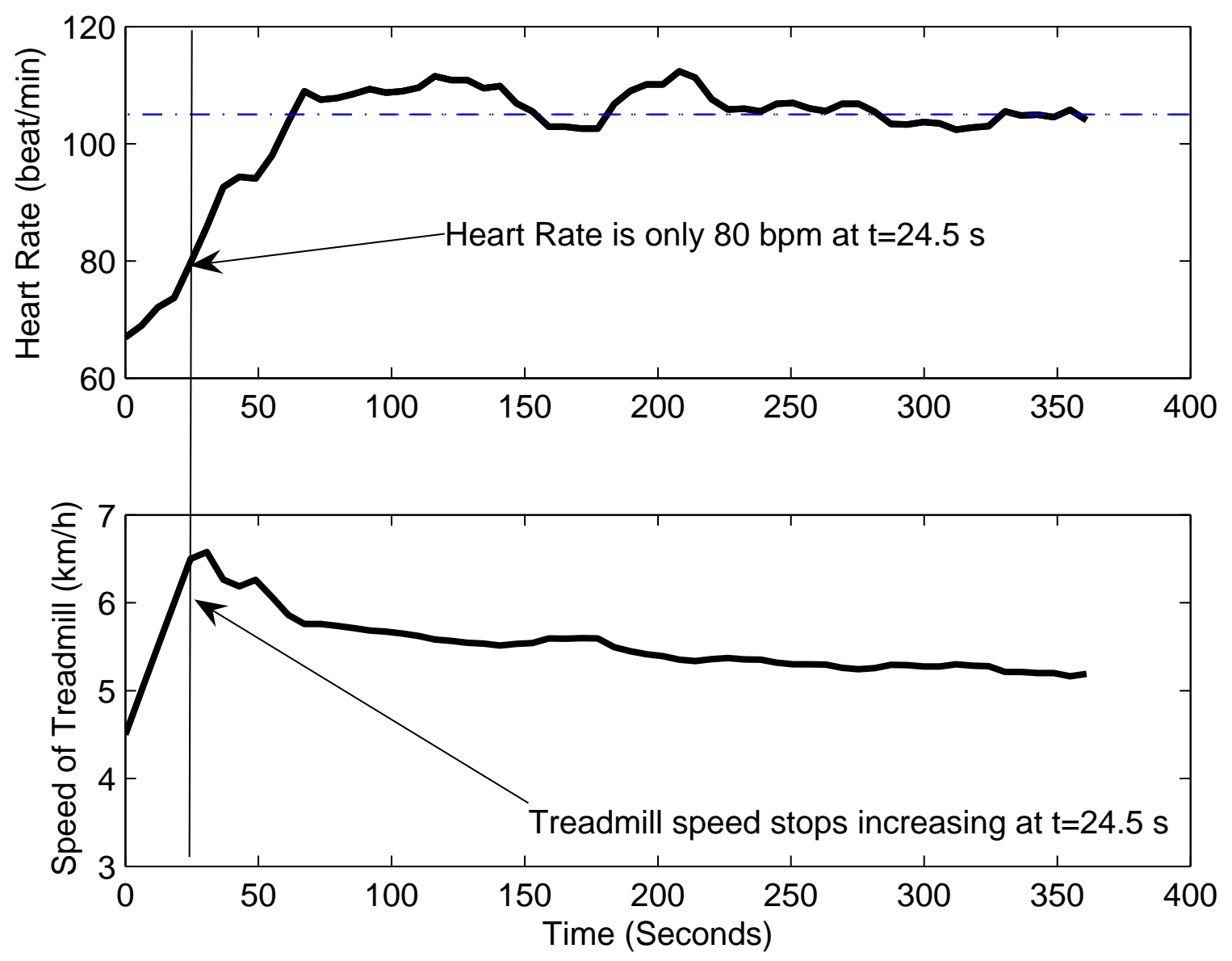

Fig. 11. A typical experimental result. 


\section{LIST OF FIGURES}

1 The proposed nonparametric identification procedure. . . . . . . . . . . . 18

2 The automated treadmill system. . . . . . . . . . . . . . . . . . . . . 19

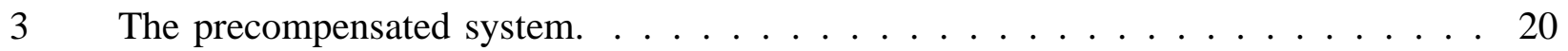

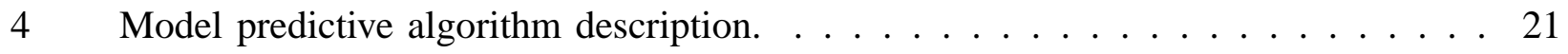

5 Inversion of nonlinearity modelling by using $\epsilon$-insensitivity SVR . . . . . . . 22

6 Correlation analysis results of six subjects. Top: Step responses of all six subjects based on correlation analysis. Bottom: Normalized step responses of all subjects. . 23

7 Step response under different acceleration constraints. Top: Heart rate sensor output (controlled output). Bottom: Treadmill speed (control effort). . . . . . . . . . . . 24

8 System response for sensor malfunction under different accelerations. . . . . . . . . 25

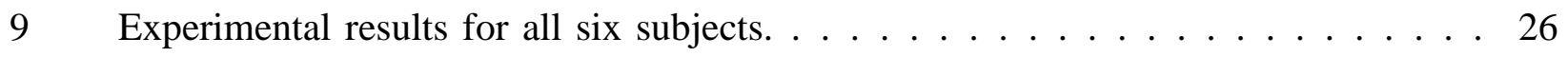

10 Comparison between proposed control approach (under different constraints) and

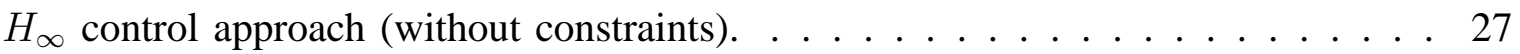

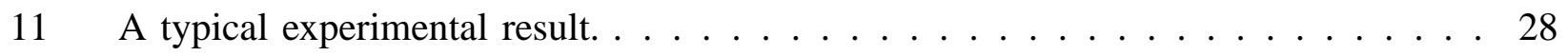

